\title{
Quantification of myocardium at risk in ST- elevation myocardial infarction: a comparison of contrast-enhanced steady- state free precession cine cardiovascular magnetic resonance with coronary angiographic jeopardy scores
}

\author{
Rodney De Palma ${ }^{* \dagger}$, Peder Sörensson ${ }^{\dagger}$, Dinos Verouhis, John Pernow and Nawzad Saleh
}

\begin{abstract}
Background: Clinical outcome following acute myocardial infarction is predicted by final infarct size evaluated in relation to left ventricular myocardium at risk (MaR). Contrast-enhanced steady-state free precession (CE-SSFP) cardiovascular magnetic resonance imaging (CMR) is not widely used for assessing MaR. Evidence of its utility compared to traditional assessment methods and as a surrogate for clinical outcome is needed.

Methods: Retrospective analysis within a study evaluating post-conditioning during ST elevation myocardial infarction (STEMI) treated with coronary intervention ( $n=78$ ). CE-SSFP post-infarction was compared with angiographic jeopardy methods. Differences and variability between CMR and angiographic methods using Bland-Altman analyses were evaluated. Clinical outcomes were compared to MaR and extent of infarction.

Results: MaR showed correlation between CE-SSFP, and both BARI and APPROACH scores of $0.83(p<0.0001)$ and 0.84 $(p<0.0001)$ respectively. Bias between CE-SSFP and BARI was 1.1\% (agreement limits -11.4 to +9.1 ). Bias between CESSFP and APPROACH was $1.2 \%$ (agreement limits -13 to +10.5 ). Inter-observer variability for the BARI score was $0.56 \pm 2$. 9; $0.42 \pm 2.1$ for the APPROACH score; $-1.4 \pm 3.1 \%$ for CE-SSFP. Intra-observer variability was $0.15 \pm 1.85$ for the BARI score; for the APPROACH score $0.19 \pm 1.6$; and for CE-SSFP $-0.58 \pm 2.9 \%$.
\end{abstract}

Conclusion: Quantification of MaR with CE-SSFP imaging following STEMI shows high correlation and low bias compared with angiographic scoring and supports its use as a reliable and practical method to determine myocardial salvage in this patient population.

Trial registration: Clinical trial registration information for the parent clinical trial:

Karolinska Clinical Trial Registration (2008)

Unique identifier: CT20080014. Registered 04 ${ }^{\text {th }}$ January 2008

Keywords: Myocardium, myocardial salvage, cardiovascular magnetic resonance imaging

\footnotetext{
* Correspondence: rodney_de_palma@icloud.com

${ }^{\dagger}$ Equal contributors

Karolinska Institutet, Department of Medicine, Unit of Cardiology, Karolinska

University Hospital, Stockholm, Sweden
} 


\section{Background}

The clinical outcome following acute ST-segment elevation myocardial infarction (STEMI) is predicted by the final infarct size $[1,2]$. This in turn is determined by a complex interplay between several factors including the duration of myocardial ischemia, the presence of collateral blood supply, reperfusion injury and left ventricular myocardium at risk (MaR). Therapies to restore myocardial perfusion and/or limit reperfusion injury, whether mechanical or pharmacological, are incompletely assessed by final absolute infarct size alone [3]. Evaluation of infarct size has to be performed in relation to the MaR. The myocardial salvage index, calculated as (MaR-final infarct size)/MaR, is a more robust surrogate for therapeutic efficacy. The reference technique for assessing the MaR is nuclear single photon emission computed tomography (SPECT) [4-6]. This imaging modality, however, is not practical in the clinical setting as it requires acute imaging when a patient may remain clinically unstable as well as two subsequent image acquisitions. Furthermore, it has relatively limited spatial resolution and involves exposure to ionizing radiation. It is therefore of interest that several studies have demonstrated that cardiovascular magnetic resonance imaging (CMR) is equivalent to SPECT at 7 days following STEMI for assessing MaR using contrastenhanced steady-state free precession cardiac magnetic resonance (CE-SSFP), or T2-weighted sequences in a single examination $[4,7-9]$.

CE-SSFP is not yet widely used for the assessment of $\mathrm{MaR}$ but has been shown to be more robust than T2weighted imaging in multi-center, multi-vendor studies $[10,11]$. Given the potential advantage of combining CE-CMR with SSFP and LGE to evaluate both MaR and final infarct size in this setting further evidence of its utility as a surrogate for clinical outcome is needed. Angiographic assessment of myocardium at risk has been shown to predict clinical outcome following myocardial infarction $[12,13]$. A comparison of CE-SSFP and such angiographic evaluation has not been undertaken previously.

\section{Aim}

The aim of the current investigation was therefore to evaluate the relationship between CE-SSFP and two validated angiographic scoring techniques for anatomical left ventricular myocardial area at risk: the BARI (Bypass Angioplasty Revascularization Investigation Myocardial Jeopardy index) score and the modified APPROACH (Alberta Provincial Project for Outcome Assessment in Coronary Heart Disease) score [12, 13]. Clinical outcome data were also evaluated in the context of $\mathrm{MaR}$ and extent of infarction.

The main hypothesis tested was that CE-SSFP provides a coherent determination of $\mathrm{MaR}$, when compared with angiographic scoring techniques.

\section{Methods}

Consecutive patients from a randomized trial evaluating post-conditioning during STEMI were analyzed retrospectively. This study was a single-center single-blinded prospective clinical trial evaluating the benefit of intracoronary balloon-mediated ischemic post-conditioning on myocardial infarct size in patients presenting with STEMI the setting of a primary percutaneous coronary intervention service with study recruitment between $1^{\text {st }}$ January 2009-31 $1^{\text {st }}$ December 2009 and has been described in detail previously [14]. In brief, eligibility consisted of a presentation within $6 \mathrm{~h}$ compatible with acute ischemia, ST elevation on a 12 lead electrocardiogram $(>0.1 \mathrm{mV}$ in two contiguous leads, $>0.2 \mathrm{mV}$ in V1-V3, or new left bundle branch block and Thrombolysis in Myocardial Infarction (TIMI) 0 flow in an identifiable infarct-related artery on invasive coronary angiography. Exclusion criteria consisted of: previous myocardial infarction including the presence of pathological Q waves on the resting electrocardiogram, surgical revascularization, cardiogenic shock, a presentation with cardiac arrest, treatment with metformin, absolute contraindication to CMR, atrial fibrillation and known renal impairment (defined as creatinine $>150$ micromol/l).

\section{Invasive coronary angiography}

Invasive coronary angiography was performed to confirm complete coronary occlusion in the infarct related artery and determine the BARI and Modified APPROACH scores. Percutaneous coronary intervention (PCI) was then performed according to local standard procedures at the discretion of the individual physician. The intervention was completed by a coronary angiographic acquisition to determine final TIMI grade flow.

Visual angiographic scoring, using the BARI and modified APPROACH systems, was carried out retrospectively using pre- and post-PCI images. Two experienced assessors, each with direct clinical experience of more than 2000 invasive procedures, and blinded to the CMR results scored the angiograms. The Rentrop scoring system was used to assess collateralization (Table 2).

\section{Post-conditioning protocol}

After invasive coronary angiography, patients were randomized to primary PCI only or primary PCI followed by post-conditioning. This was performed by intracoronary occlusion using the PCI balloon to a pressure of 2-4 atmospheres for four cycles of $60 \mathrm{~s}$ starting $60 \mathrm{~s}$ after the initial reperfusion [14].

\section{Cardiovascular magnetic resonance imaging}

A standard CMR was scheduled between 5-7 days after admission. These investigations were performed in the supine position with an eight-channel cardiac coil by 
means of a 1.5 Tesla system (Signa Excite TwinSpeed, General Electric Healthcare, Waukesha, Wisconsin, USA) during vector-ECG monitoring. Intravenous gadolinium-DTPA chelated contrast $(0.2 \mathrm{mmol} / \mathrm{kg}$; Omniscan, GE Healthcare) was administered before positioning the patient in the scanner. The image protocol included scout images, localization of the short axis and then covering the whole left ventricle (LV) with cine balanced steady-state free precession (SSFP) images retrospectively-gated. Baseline images were acquired $5 \mathrm{~min}$ after contrast administration. The following parameters were used; SSFP (echo time (TE) 1.58 milliseconds (ms), repetition time (TR) $3.61 \mathrm{~ms}$, flip angle $60^{\circ}, 25$ phases, 8 millimetres (mm) slice, no inter-slice gap, matrix 226x226). Late gadolinium enhancement (LGE) images were acquired 15-20 min after contrast injection using an inversion recovery gradient echo sequence (TE $3.3 \mathrm{~ms}$, TR $7.0 \mathrm{~ms}$, inversion time $180-250 \mathrm{~ms}$ to null the myocardium, $8 \mathrm{~mm}$ slice, no inter-slice gap, matrix 256x192) and the same slice orientation as cine SSFP images $[15,16]$.

Cardiac triggering was set for diastole to reduce motion artifacts. Each slice was obtained during end-expiratory breath holding. Two-, three- and four-chamber views were also obtained to confirm the findings.

CMR images were analyzed off-line at a later session using freely available segmentation software (Segment V.1.8 R0857; http://segment.heiberg.se/) [17]. Enddiastolic and end- systolic volumes, ejection fraction, stroke volume and left ventricular volume were calculated on cine SSFP sequences. Infarct size was quantified using an automated quantification method that has been validated ex vivo and in vivo in which account is made for partial volume effects $[18,19]$. The extent of infarction is expressed both in absolute mass and as the percentage of MaR with LGE.

MaR determined from CE-SSFP was assessed according to previously described methodology [9]. Endo- and epicardial borders were manually traced in all shortaxis slices in both end-diastole and end-systole followed by manual delineation of the hyper-intense regions in end-diastole and end-systole, by one experienced observer (level 2 CMR certified and with 12 years of CMR experience) blinded to the LGE images and angiographic scores (Fig. 1). A second experienced observer (level 3 CMR certified and with 20 years of CMR experience) evaluated twelve patients for intra-observer variability. Hypo-intense myocardium within the area of increased signal intensity was regarded as microvascular obstruction and was included in the MaR. The values of $\mathrm{MaR}$ in end-diastole and end-systole were averaged and expressed as a percentage of LV mass, (Fig. 1 and Additional file 1: movie1).

\section{BARI score}

Left ventricular MaR was calculated by grading all terminating arteries. All branches were scored as 3, 2, 1 or 0 points, corresponding to, large, medium, small or absent respectively. The ventricular base to apex distance, approximated from the coronary angiogram, provided the basis for assessing the relative distribution of coronary branches. Branches were considered large if their length exceeded two- thirds of the distance from base to apex, medium if one-third to two-thirds the distance, small if less than one-third of the distance and absent if less than one-fifth of the distance from base to apex [12]. The risk score was calculated as a percentage of the left ventricular myocardial volume; by dividing summed scores of a jeopardized territory by the total score of the entire left ventricle.

\section{Modified APPROACH score}

This score, which is based on pathological and necropsy studies $[20,21]$, is derived from a template that

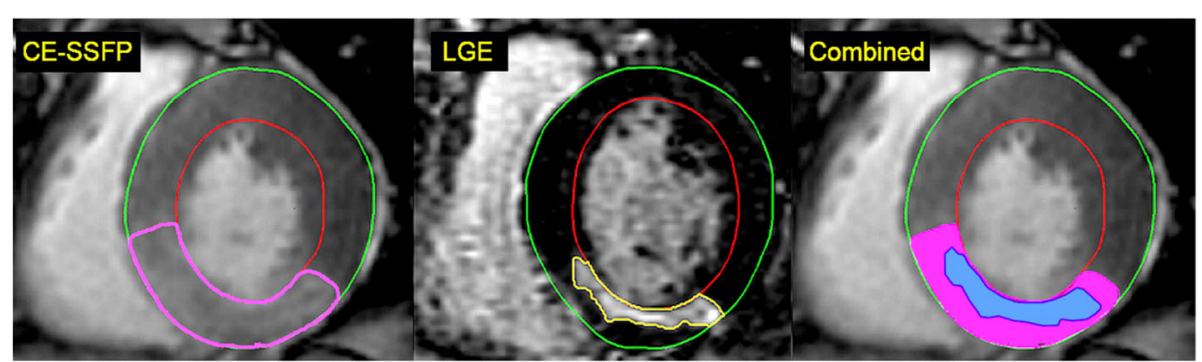

Fig. 1 Example of MaR and myocardial salvage index using contrast-enhanced SSFP magnetic resonance imaging. Corresponding left ventricular short axis views from a patient with an inferior STEMI caused by a right coronary artery occlusion. The epicardium is delineated by a green border, and the endocardium by a red border. The myocardium at risk (MaR) in the inferior segment is determined by area of early gadolinium enhancement on SSFP (delineated by a pink border in the panel to the left). The size of infarction in the inferior segment is determined by the area of late gadolinium enhancement delineated by a yellow border in the middle panel. Automatic computer-aided superimposition of the infarcted area and the MaR area can be used to calculate the myocardial salvage index (as a ratio of the two areas, 1-scar area/MaR), panel to the right. MaR= myocardium at risk; CE-SSFP = contrast-enhanced steady-state free precession; STEMI = ST-elevation myocardial infarction; LGE = late gadolinium enhancement 
considers the culprit artery, dominance and lesion location (proximal or mid-vessel) together with the size (small, medium or large) of the remaining major nonculprit epicardial vessel [13]. The score in the template represents the percentage of LV MaR.

\section{Statistical methods}

Data management and analyses were performed using EXCEL version 14.4.3 (Microsoft Corporation, Redmond, Wisconsin, USA) and GraphPad Prism V.5.00 (GraphPad Software, San Diego, California, USA).

Continuous data are presented as means with standard deviations (SD). Categorical data are presented as frequencies with percentages or medians with interquartile ranges (IQR).

The endpoint was the mean bias between the angiographic scores and MaR derived from the CE-SSFP images using Bland-Altman plots and expressed as mean and limits of agreement $(2 \times \mathrm{SD})$.

Pearson's correlation coefficient was also used to evaluate the relationship between CE-SSFP and the angiographic risk scores.

Intra- and inter-observer variability was calculated as the SD of the difference between two calculations divided by the mean of the two observers and expressed as the mean $+/-\mathrm{SD}$.

Statistical significance was accepted as $p<0.05$.

\section{Results}

A total of 89 patients out of a screened cohort of 795 presented with acute STEMI and TIMI 0 flow in the infarct-related artery and were eligible. Those not fulfilling the inclusion criteria were excluded prior to randomization $(n=706)$. Eleven patients were excluded following randomization due to poor CMR image quality, not fulfilling the post-conditioning protocol, or inhospital death prior to imaging.

Those finally selected comprised 78 patients who underwent CMR examination at a median of 7 days (IQR 6-9) following admission.

Baseline clinical characteristics show the group to be young and predominantly male. Smoking and hypertension were present in a substantial proportion of patients but diabetes mellitus and previous symptoms of ischemic heart disease were infrequent.

The absence of prior symptomatic ischemic heart disease is also reflected by the small proportion of patients on cardiovascular preventive therapies prior to admission (Table 1).

In $35 \%$ of patients the culprit artery was the left anterior descending artery, in $57 \%$ of patients it was the right coronary artery, and in $8 \%$ it was the circumflex artery. No patients presented with a left main occlusion. Collateral circulation was visible in $18 \%$ (Table 2).
Table 1 Clinical characteristics of the cohort

\begin{tabular}{ll}
\hline Variables & $N=78$ \\
\hline On admission & \\
Age, years (SD) & $62 \pm 11$ \\
Male sex, n (\%) & $65(83)$ \\
Body mass index, kg/m² (IQR) & $27(25-30)$ \\
Ischemia time, minutes (IQR) & $173(140-239)$ \\
Current smoker, n (\%) & $24(31)$ \\
Hypertension, n (\%) & $18(23)$ \\
Dyslipidemia, n (\%) & $6(8)$ \\
Previous angina, n (\%) & $9(12)$ \\
Previous known diabetes mellitus, n (\%) & $2(3)$ \\
Treatment on admission & \\
Aspirin, $\mathrm{n}(\%)$ & $5(6)$ \\
Beta-blocker, n (\%) & $6(8)$ \\
ACE/ARB, n (\%) & $7(9)$ \\
Statin & $6(8)$ \\
Treatment at discharge & \\
Aspirin, n (\%) & $77(99)$ \\
Clopidogrel, n (\%) & $78(100)$ \\
Beta-blocker, n (\%) & $77(99)$ \\
ACE/ARB, n (\%) & $44(56)$ \\
Statin, n (\%) & $76(97)$ \\
\hline Data are presed &
\end{tabular}

Data are presented as number of patients and percentage in brackets for dichotomous variables or median and interquartile range. $A C E$ angiotensin converting enzyme inhibitor, $A R B$ angiotensin receptor blocker, $I Q R$ inter-quartile range

Table 2 Angiographic characteristics of the cohort

\begin{tabular}{ll}
\hline Variables & $N=78$ \\
\hline Infarct related artery & $27(35)$ \\
LAD & $6(8)$ \\
LCX & $45(57)$ \\
RCA & \\
Disease pattern & $49(63)$ \\
One-vessel disease & $22(28)$ \\
Two-vessel disease & $7(9)$ \\
Three-vessel disease & $69(88)$ \\
TIMl grade 3 flow after PCl & $64(82)$ \\
Collateral flow (Rentrop grade 0 or 1) & $14(18)$ \\
Collateral flow (Rentrop grade 2 or 3)
\end{tabular}

Data are presented as number of patients and percentage in brackets for dichotomous variables. $L A D$ left anterior descending coronary artery, $R C A$ right coronary artery, $L C X$ left circumflex coronary artery, TIMI Thrombolysis In Myocardial Infarction, $\mathrm{PCl}$ percutaneous coronary intervention

Rentrop grade $0=$ no filling of distal infarct vessel by collateral vessels Rentrop grade 1 = filling of distal infarct vessel side branches only by collateral vessels

Rentrop grade 2 = partial filling of distal infarct main vessel by collateral vessels

Rentrop grade $3=$ complete filling of distal infarct main vessel by collateral vessels 
There were no significant differences in the percentage $\mathrm{MaR}$ based on culprit vessel or background coronary disease pattern between the methods (Table 3).

All patients had a sufficient number and quality of angiographic projections to determine the culprit and non-culprit coronary anatomy.

\section{Relationship between CE-SSFP and angiographic scores}

There was a good linear correlation between the BARI and modified APPROACH scores and that derived from CMR $(0.83 p<0.0001$ and 0.84 respectively, $p<0.0001)$ (Figs. 2 and 3). Bland-Altman analysis demonstrated that there was good agreement of the size of the MaR between CE-SSFP and angiographic scores (mean of $1.1 \%+/-5.2$ with limits of agreement -11.4 to +9.1 , and $1.2 \%+/-6.0$ with limits of agreement -13 to +10.5 , for the BARI and Modified APPROACH scores respectively) (Figs. 2 and 3).

\section{Reproducibility amongst observers}

The angiographic and CMR estimated MaR did not differ between techniques (Table 3). The inter-observer variability showed a mean difference $0.56 \pm 2.9 \%$ for the BARI score $(n=12,95 \% \mathrm{CI}=0.84$ to 0.28$), 0.42 \pm 2.1 \%$ for the Modified APPROACH score $(n=12,95 \% \mathrm{CI}=$ 0.70 to 0.14$)$ and $-1.4 \pm 3.1 \%$ for the CE-SSFP technique $(n=12,95 \% \mathrm{Ci}=-0.36$ to -2.44$))$. The intra-observer variability showed a mean difference $0.15 \pm 1.85 \%$ for the BARI score $(95 \% \mathrm{CI}=0.35$ to -0.20$), 0.19 \pm 1.6 \%$ for the

Table 3 Myocardium at risk by method used and angiographic characteristics

\begin{tabular}{llll}
\hline Variables & CMR & BARI & APPROACH \\
\hline $\begin{array}{l}\text { Total MaR } \\
\text { Infarct related artery }\end{array}$ & $30(7.7-56.9)$ & $28.5(12-50)$ & $28.0(12-48)$ \\
LAD & $42(32-49)$ & $41(33-46)$ & $44(30-47)$ \\
LCX & $40(35-46)$ & $34(32-37)$ & $30(28-40)$ \\
RCA & $26(22-32)$ & $27(22-29)$ & $28(24-28)$ \\
Disease pattern & & & \\
One-vessel disease & $31(25-44)$ & $29(27-40)$ & $28(27-44)$ \\
Two-vessel disease & $31(25-39)$ & $30(25-34)$ & $28(28-28)$ \\
Three-vessel disease & $24(22-32)$ & $22(22-26)$ & $24(22-28)$ \\
Collateral flow grade 0 & $31(24-43)$ & $30(25-39)$ & $28(27-44)$ \\
Collateral flow grade 1 & $31(27-39)$ & $28(27-36)$ & $28(27-29)$ \\
Collateral flow grade 2 or 3 & $21(7-26)$ & $27(22-29)$ & $28(22-28)$ \\
\hline
\end{tabular}

Data on percentage myocardium at risk are presented as median and interquartile range (in brackets). There were no significant differences between the methods according to infarct-related artery or underlying coronary disease pattern. $L A D$ left anterior descending coronary artery, $R C A$ right coronary artery, $L C X$ left circumflex coronary artery, $C M R$ cardiac magnetic resonance, MaR myocardium at risk. There were no statistically significant differences between the MaR and collateralization for each of the angiographic techniques and CMR. Friedman test $p=0.157$ and 0.06 respectively for collaterals 0 vs 2 and 3

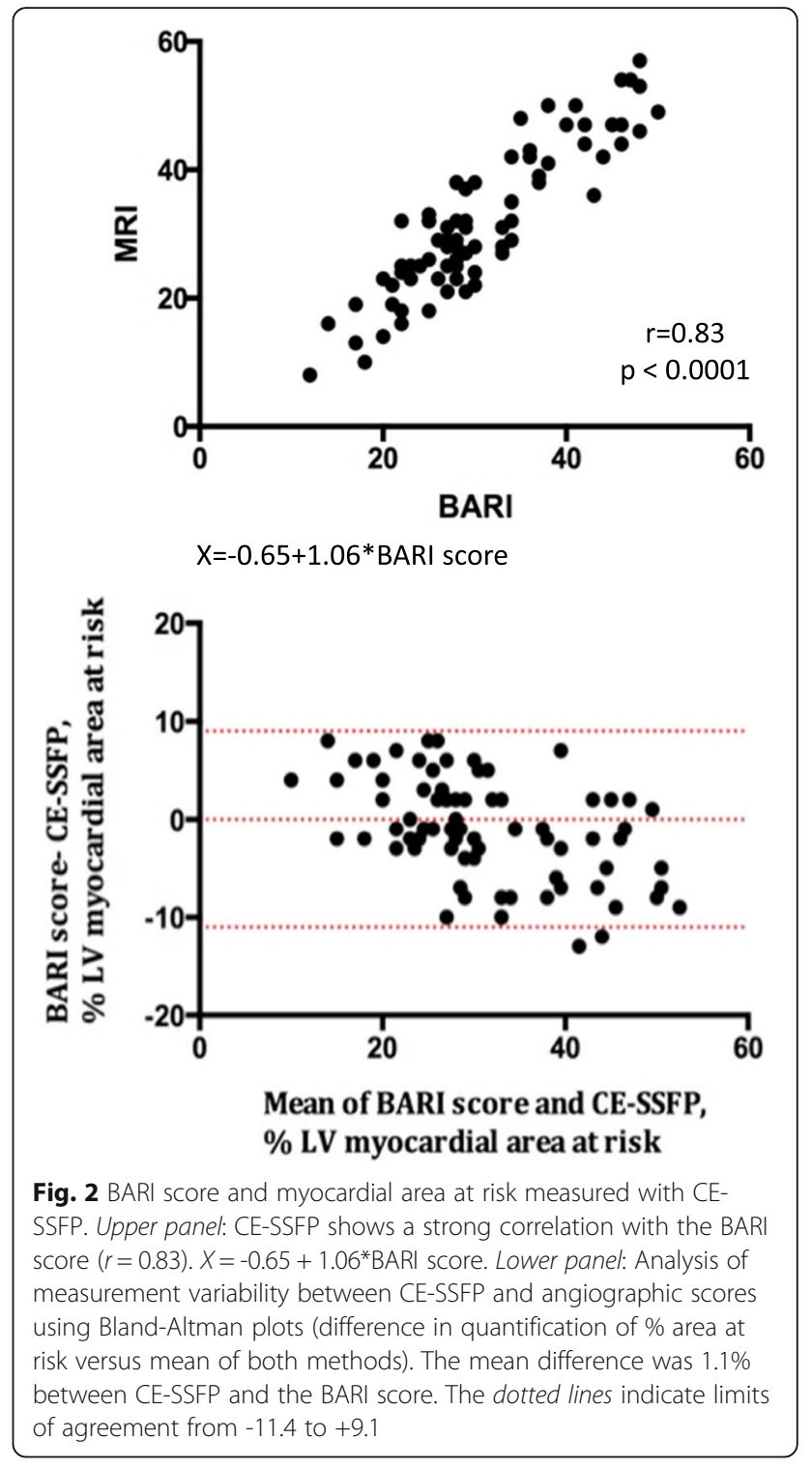

Modified APPROACH score $(95 \% \mathrm{CI}=0.41$ to -0.03$)$ and for CE-SSFP $-0.58 \pm 2.9 \%(95 \% \mathrm{CI}=-0.04$ to -1.12$)$

\section{Discussion}

The main finding is that contrast enhanced CE-SSFP $\mathrm{CMR}$ evaluation of MaR correlates well with angiographic jeopardy scores validated previously as surrogates for clinical outcome [22]. The bias was small with very mild overestimation, 1.1 and $1.2 \%$ for the BARI and Modified APPROACH scores respectively. The limits of agreement between the CMR and angiographic measurements were -11.4 to +9.1 , and -13 to +10.5 for the BARI and Modified APPROACH scores respectively. There was consistent variability across the range of MaR measured.

Final infarct size in acute STEMI is a key determinant for clinical outcome and has to be determined in relation to MaR [1, 2]. Due to the large variability in 


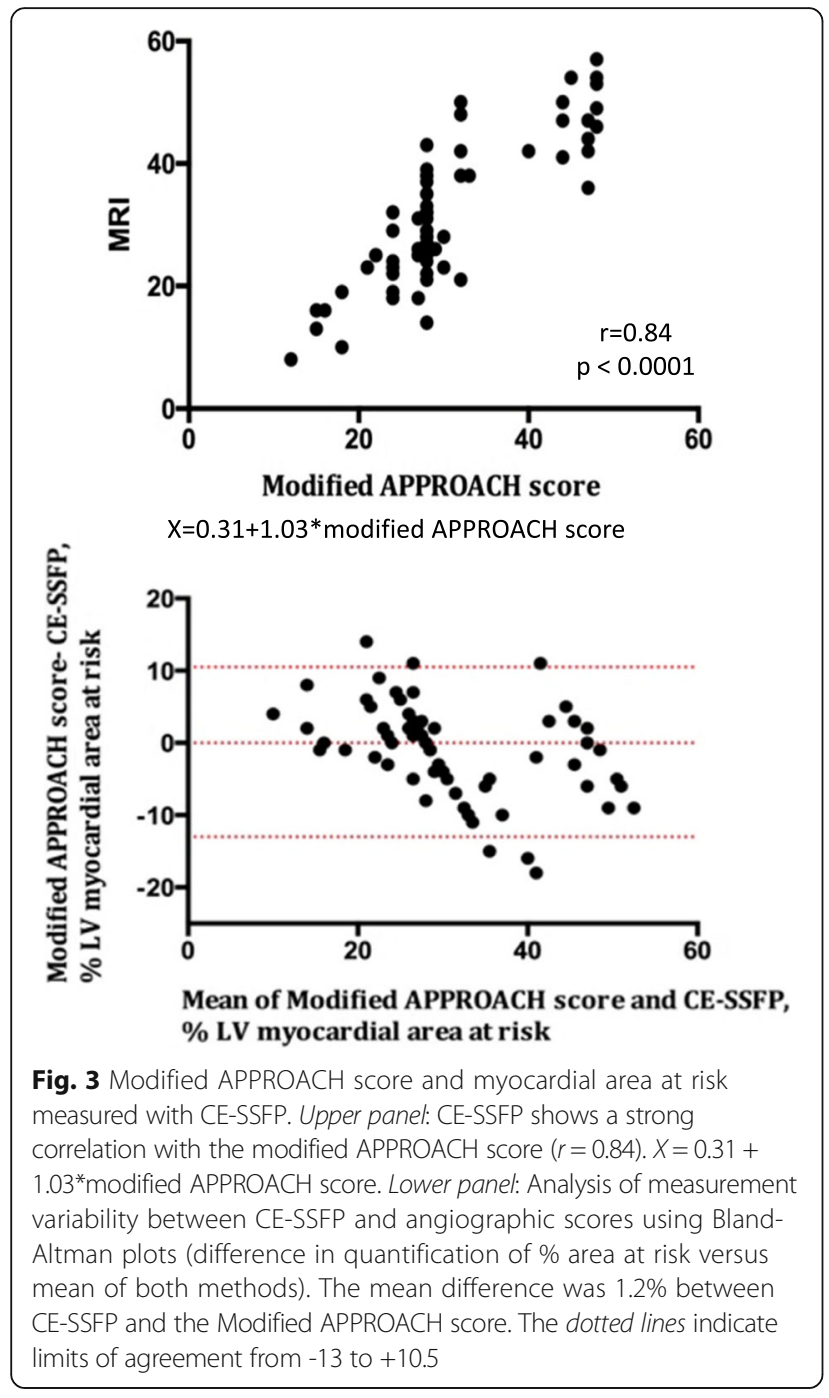

absolute infarct size, very large study groups are needed to achieve adequate power in clinical studies having infarct size as an end-point. Myocardial salvage is a pragmatic surrogate measurement for therapeutic efficacy of interventions aiming at limiting infarct size since it normalizes the infarct size to MaR. Thus, the use of CMR and myocardial salvage index (rather than myocardial infarction size alone) substantially reduces the study group sizes needed to evaluate efficacy in clinical trials [23]. It is therefore of utmost importance to develop feasible methods that accurately determine MaR in the clinical setting. Furthermore, the evaluation of the peri-infarct zone, which is also a feature of CMR evaluation, can add incremental prognostic value [24].

\section{Cardiovascular magnetic resonance evaluation of myocardium at risk}

CMR is increasingly recognized as equivalent to nuclearbased techniques for determining the MaR whilst also having inherent practical advantages. Several CMR sequences have been evaluated in this context including CESSFP $[9,11,25,26]$. In 2012, Moral et al, compared BARI and APPROACH scores with T2-STIR and infarct endocardial surface area methods. They reported a good correlation between the different angiographic scores and their CMR-sequence [27]. The data presented here, as far as we are aware, represent the first comparison of CE-SSFP and angiographic jeopardy scores in the literature.

The advantage of this method is that it can be easily added to current standard clinical protocols, in scanners from all major vendors, simply by administering a single dose of gadolinium contrast agent before acquiring short-axis cine images. This shortens the CMR protocol in the scanner and the evaluation time after the examination since the same images can be used both for MaR determination and the evaluation of functional parameters. Unstable patients may also benefit from shorter CMR protocols.

Bright-blood T2-weighted sequences have been developed to increase the diagnostic accuracy of T2 CMR for depicting edema $[28,29]$. One such sequence (ACUT2E) was more accurate for the determination of $\mathrm{MaR}$ and myocardial salvage than was dark-blood T2-weighted sequences, which results in underestimation of MaR [30]. Thus, CE-SSFP might be more accurate than dark-blood T2 since CE-SSFP showed larger MaR in a study by Ubachs et al [8]. Several limitations of different T2weighted imaging protocols include a lack of consensus on the optimal quantification method, signal intensity variability within each slice and susceptibility to motion artefacts [7, 31, 32]. Newly developed T1- or T2-mapping techniques may improve accuracy considerably on these limitations when using motion correction and an absolute threshold for edema, [33-35] but further standardization and validation are still needed.

In patients, therefore, where there is no contraindication to gadolinium, the CE-SSFP may nevertheless still remain a more practical method to apply, particularly in timeconstrained contemporary clinical working environments. Furthermore, the multi-phase acquisition of SSFP throughout the cardiac cycle allows for more robust delineation of endocardial and epicardial borders, less image artefacts, better spatial resolution, and as a consequence better inclusion rates of patients in clinical studies.

The mechanism for contrast-enhancement of the MaR during SSFP cannot be explained fully. The contrast in SSFP images is dependent on the sequence T2/T1 ratio. $\mathrm{T} 2$ relaxation times are based on the increase in tissue water content and mobility, which is a characteristic of MaR [36]. When using a gadolinium-based contrast agent, the T1 for the surrounding tissue is shortened. This is also the rationale for infarct visualization in T1weighted inversion-recovery LGE imaging since the 
contrast agent distributes to the extracellular space. Here the concentration of an extracellular gadolinium-based contrast agent is increased due to an increased distribution volume in myocardium injured by ischemia [37-40]. It has been shown that even reversibly injured myocardium within the MaR has an increased distribution volume in the acute phase after an ischemic episode [41, 42]. The T2/T1 ratio in the entire MaR, including both reversible and irreversibly injured myocardium, is affected by the presence of gadolinium. This might therefore explain the increased signal intensity in the MaR seen by CE-SSFP.

There is an ongoing scientific discourse regarding the conditioning treatments of reperfusion injury and their effect on myocardial edema. Two recent studies [43, 44] have shown reduced MaR in the active group (local post-conditioning and remote pre-conditioning) of patients compared to controls, but the protocols and sequences used were not the same as the one used in our study. In addition, these studies used T2-STIR and T2mapping respectively. The post-conditioning protocol in theory could have influenced the $\mathrm{MaR}$ calculated by $\mathrm{CE}$ SSFP in the present study. The final infarct size and MaR, however, as judged by the extent of CE-SSFP and LGE was similar in both the control arm and postconditioning arm. Furthermore, there were no differences in clinical outcomes between the groups. These observations suggest that any contribution of the treatment protocol to the MaR was minimal.

Collateral supply to an infarcted territory could also influence the final infarct size and MaR. Although there was no statistically significant difference between each of the angiographic quantification methods and the MaR for the extent of collateralization, there was a trend to significance with CE-SSFP evaluation $(p=0.157$ and 0.06 respectively for Rentrop collaterals 0 vs a composite of Rentrop 2 and 3). This points to the intuitive consideration that CE-SSFP may have an added benefit as it takes the collateral supply into account whereas the rigid angiographic scoring techniques do not.

\section{Angiographic evaluation of myocardium at risk}

The modified-APPROACH score is based on pathological and necropsy studies and is derived from a template that considers the culprit artery, dominance and lesion location (proximal or mid-vessel) together with the size (small, medium or large) of the remaining major non-culprit epicardial vessel. The score in the template represents the percentage of left ventricle at risk.

The BARI score bases the anatomical area at risk and the length and caliber of the epicardial coronaries and has been validated against post-mortem histological studies [21].
Both scores have been validated with respect to adverse clinical outcome in a large population-based cohort (20,067 patients) undergoing PCI with similar cstatistics for predicting one-year mortality, when added to simple baseline characteristics, of 0.85 and 0.84 for the APPROACH and BARI scores respectively [22]. For both these angiographic scores their validity is based predominantly on left anterior descending and right coronary artery infarctions. Challenges in the electrocardiographic diagnosis of circumflex occlusion leads to under-representation of this category of infarction [45].

The BARI score is more precise than the modified APPROACH score but is also more time-consuming. The modified APPROACH score on the other hand is subject to more observer variability. This study also reflects the broader variability of the latter compared to the former on Bland-Altman analysis. Whilst the BARI score takes into consideration stenotic lesions $>50 \%$ and the Modified APPROACH score considers a lesion $>70 \%$ as being significant, in the context of acute STEMI this difference in scoring is not manifest as the culprit vessel is occluded. The Modified APPROACH score is based on fixed assumptions of the myocardium at risk subtended by the location of a lesion in the coronary artery and hence clustering of high, intermediate and lower values can be seen when compared with CE-SSFP. There was no difference, however, in clinical outcomes and the absolute extent of infarction or in relation to MaR as estimated by either angiographic score or CE-SSFP. In addition, there was no difference in extent of infarction when adjusted for angiographically-evident collateralization. These data thus indicate that despite the precision of the BARI score, both angiographic techniques for estimating the size of MaR appeared to be clinically equivalent in this study.

Angiographic risk scores have been used to validate single-photon emission computed tomography as well as T2 weighted CMR $[7,46]$.

This study was not able to disprove the hypothesis that there was no difference between angiographic scoring and CE-SSFP for the assessment of MaR, hence validating its use for this purpose. The close correlation between the angiographic and CE-SSFP measurements of myocardial area at risk adds further support that CE-SSFP may be a useful tool in studies investigating interventional, pharmacological and cardioprotective therapies in STEMI. These data and the practicality of adding early contrast enhancement to standard SSFP protocols, makes this a widely applicable method.

\section{Limitations}

The study was a single center retrospective analysis and thus subject to selection bias that may limit its external validity. The study was a post-hoc analysis from a parent 
study that was powered for clinical endpoints and so the analysis presented here is open to type 2 error. The results may have been influenced by the post-conditioning protocol and presence of collateral blood supply. The post-conditioning protocol, however, did not affect the overall infarct size. Furthermore, as infarct size was similar, an analysis on the differential predictive capacity for the techniques and infarct size based on therapy could not be performed

The angiographic jeopardy scores are based on anatomical assumptions and the relationships between these and other scoring systems in predicting clinical outcome is unclear.

No semi-quantitative method was used to determine MaR with the CE-SSFP sequence since the signal intensity difference between remote myocardium and MaR is small and makes it difficult to choose a fixed threshold value. Nevertheless, the inter-observer variability was excellent between the image readers. However, a new freely available semi-automatic software for estimating MaR using CE-SSFP has recently been validated and published [19]. This program can be used in all major vendors and can further decrease variability as well as increase the accuracy in estimating MaR for future reperfusion studies.

\section{Conclusion}

Quantification of MaR with contrast-enhanced SSFP imaging following acute STEMI shows high correlation and low bias compared with angiographic scoring and further supports its use as a reliable and practical method to determine myocardial salvage in this patient population.

\section{Additional file}

Additional file 1: Movie 1. Contrast-enhanced SSFP cine loop of a full base-to-apex coverage short-axis slice set, with each slice moving through the full systolic and diastolic cycle, used to quantify MaR (corresponds to Fig. 1). (MOV 803 kb)

\footnotetext{
Abbreviations

ACE: angiotensin converting enzyme inhibitor; APPROACH: Alberta Provincial Project for Outcome Assessment in Coronary Heart Disease; ARB: angiotensin receptor blocker; BARI: Bypass Angioplasty Revascularization Investigation Myocardial Jeopardy index; CE-SSFP: contrast-enhanced steady-state free precession; Cl: confidence interval; CMR: cardiovascular magnetic resonance imaging; IQR: interquartile ranges; LAD: left anterior descending coronary artery; LCx: left circumflex coronary artery; LGE: late gadolinium enhancement; LV: left ventricle; MACCE: major adverse cardiovascular and cerebral events; MaR: myocardium at risk; PCl: percutaneous coronary intervention; RCA: right coronary artery; SD: standard deviation; SPECT: nuclear single photon emission computed tomography; STEMI: ST elevation myocardial infarction; TE: echo time; TIMI: Thrombolysis in Myocardial Infarction; TR: repetition time
}

\section{Funding}

Not applicable for this study. The parent trial was funded originally by the Swedish Heart-Lung Foundation, Swedish Research Council of Medicine (10857, 14231), Stockholm County Council.

\section{Availability of data and materials}

Available upon request.

\begin{abstract}
Authors' contributions
RDP data collection, data analysis and interpretation, drafting the article, critical revision of the article, final approval of the version to be published. PS data analysis and interpretation, drafting the article, critical revision of the article, final approval of the version to be published. DV critical revision of the article, final approval of the version to be published. JP Conception and design of the work, data analysis and interpretation, drafting the article, critical revision of the article, final approval of the version to be published. NS and design of the work, data collection, data analysis and interpretation, drafting the article, critical revision of the article, final approval of the version to be published.
\end{abstract}

\section{Authors' information}

Not applicable.

\section{Competing interests}

The authors declare that they have no competing interests.

\section{Consent for publication}

Not applicable.

\section{Ethics approval and consent to participate}

The study was performed according to the Declaration of Helsinki and good clinical practice. Written informed consent for the parent trial was given by all patients. The parent trial and amendment for this study analysis was approved by the local ethics committee at the Karolinska Institutet. Original ethics parent trial identifier: 2006/733-31.

Amendment for this study: 2016/862-32.

\section{Publisher's Note}

Springer Nature remains neutral with regard to jurisdictional claims in published maps and institutional affiliations.

Received: 29 November 2016 Accepted: 3 May 2017

Published online: 27 July 2017

\section{References}

1. Braunwald E. Myocardial reperfusion, limitation of infarct size, reduction of left ventricular dysfunction, and improved survival. Should the paradigm be expanded? Circulation. 1989;79:441-4.

2. Fibrinolytic Therapy Trialists' (FTT) Collaborative Group. Indications for fibrinolytic therapy in suspected acute myocardial infarction: collaborative overview of early mortality and major morbidity results from all randomised trials of more than 1000 patients. Lancet. 1994;343:311-22.

3. Wang QD, Pernow J, Sjoquist PO, Ryden L. Pharmacological possibilities for protection against myocardial reperfusion injury. Cardiovasc Res. 2002;55:25-37.

4. Gibbons RJ, Verani MS, Behrenbeck T, Pellikka PA, O'Connor MK, Mahmarian $\mathrm{JJ}$, Chesebro JH, Wackers FJ. Feasibility of tomographic 99mTc-hexakis-2methoxy-2-methylpropyl-isonitrile imaging for the assessment of myocardial area at risk and the effect of treatment in acute myocardial infarction. Circulation. 1989:80:1277-86.

5. De Coster PM, Wijns W, Cauwe F, Robert A, Beckers C, Melin JA. Area-at-risk determination by technetium-99 m-hexakis-2-methoxyisobutyl isonitrile in experimental reperfused myocardial infarction. Circulation. 1990;82:2152-62.

6. Sinusas AJ, Trautman KA, Bergin JD, Watson DD, Ruiz M, Smith WH, Beller GA. Quantification of area at risk during coronary occlusion and degree of myocardial salvage after reperfusion with technetium-99 m methoxyisobutyl isonitrile. Circulation. 1990;82:1424-37.

7. Fuernau G, Eitel I, Franke V, Hildebrandt L, Meissner J, de Waha S, Lurz P, Gutberlet M, Desch S, Schuler G, Thiele H. Myocardium at risk in ST-segment elevation myocardial infarction comparison of T2-weighted edema imaging 
with the MR-assessed endocardial surface area and validation against angiographic scoring. JACC Cardiovasc Imaging. 2011;4:967-76.

8. Ubachs JF, Sörensson P, Engblom H, Carlsson M, Jovinge S, Pernow J, Arheden $\mathrm{H}$. Myocardium at risk by magnetic resonance imaging: head-tohead comparison of T2-weighted imaging and contrast-enhanced steadystate free precession. Eur Heart J Cardiovasc Imaging. 2012;13:1008-15.

9. Sörensson P, Heiberg E, Saleh N, Bouvier F, Caidahl K, Tornvall P, Rydén L, Pernow J, Arheden $\mathrm{H}$. Assessment of myocardium at risk with contrast enhanced steady-state free precession cine cardiovascular magnetic resonance compared to single-photon emission computed tomography. J Cardiovasc Magn Reson. 2010;12:25.

10. Goldfarb JW, Arnold S, Han J. Recent myocardial infarction: assessment with unenhanced T1-weighted MR imaging. Radiology. 2007;245:245-50.

11. Nordlund D, Klug G, Heiberg E, Koul S, Larsen TH, Metzler B, Erlinge D, Atar D, Carlsson M, Engblom H, Arheden H. Performance of contrast enhanced SSFP and T2-weighted imaging for determining myocardium at risk in a multi-vendor, multi-center setting- data from the MITOCARE and CHILL-MI trials. J Cardiovasc Magn Reson. 2015;17 Suppl 1:194.

12. Alderman EL, Stadius M. The angiographic definitions of the Bypass Angioplasty Revascularization Investigation. Coron Artery Dis. 1992;3:1 189-207.

13. Ortiz-Perez JT, Meyers SN, Lee DC, Kansal P, Klocke FJ, Holly TA, Davidson CJ, Bonow RO, Wu E. Angiographic estimates of myocardium at risk during acute myocardial infarction: validation study using cardiac magnetic resonance imaging. Eur Heart J. 2007;28:1750-8.

14. Sörensson $P$, Saleh $N$, Bouvier $F$, Böhm F, Settergren $M$, Caidahl $K$, Tornvall $P$, Arheden $\mathrm{H}$, Rydén L, Pernow J. Effect of post-conditioning on infarct size in patients with ST elevation myocardial infarction. Heart. 2010;96:1710-5.

15. Kim RJ, Fieno DS, Parrish TB, Harris K, Chen EL, Simonetti O, Bundy J, Finn JP, Klocke FJ, Judd RM. Relationship of MRI delayed contrast enhancement to irreversible injury, infarct age, and contractile function. Circulation. 1999. 100:1992-2002.

16. Simonetti OP, Kim RJ, Fieno DS, Hillenbrand HB, Wu E, Bundy JM, Finn JP, Judd RM. An improved MR imaging technique for the visualization of myocardial infarction. Radiology. 2001;218:215-23.

17. Heiberg E, Sjögren J, Ugander M, Carlsson M, Engblom H, Arheden H. Design and validation of segment - a freely available software for cardiovascular image analysis. BMC Med Imaging. 2010;10:1.

18. Heiberg E, Ugander M, Engblom H, Götberg M, Olivecrona GK, Erlinge D, Arheden $\mathrm{H}$. Automated quantification of myocardial infarction from MR images by accounting for partial volume effects: animal, phantom, and human study. Radiology. 2008;246:581-8.

19. Tufvesson J, Carlsson M, Aletras AH, Engblom H, Deux JF, Koul S, Sörensson P, Pernow J, Atar D, Erlinge D, Arheden $\mathrm{H}$, Heiberg E. Automatic segmentation of myocardium at risk from contrast enhanced SSFP CMR validation against expert readers and SPECT. BMC Med Imaging. 2016;16:19.

20. Brandt PW, Partridge JB, Wattie WJ. Coronary arteriography; method of presentation of the arteriogram report and a scoring system. Clin Radiol. 1977;28:361-5.

21. Kalbfleisch $\mathrm{H}$, Hort W. Quantitative study on the size of coronary artery supplying areas postmortem. Am Heart J. 1977;94:183-8.

22. Graham MM, Faris PD, Ghali WA, Galbraith PD, Norris CM, Badry JT, Mitchell LB, Curtis MJ, Knudtson ML. (Alberta Provincial Project for Outcome Assessment in Coronary Heart Disease) Validation of three myocardial jeopardy scores in a population-based cardiac catheterization cohort. Am Heart J. 2001:142:254-61.

23. Engblom H, Heiberg E, Jensen SE, Nordrehaug JE, Dubois-Randé J-L, Halvorsen S, Koul S, Erlinge D, Atar D, Carlsson M, Arhedren H. Design of clinical cardioprotection trials using CMR: impact of myocardial salvage index and a narrow inclusion window on sample size. J Cardiovasc Magn Reson. 2015;17 Suppl 1:90.

24. Shayne AJ, Brown KA, Gupta SN, Chan CW, Luu TM, Di Carli MF, Reynolds HG, Stevenson WG, Kwong RY. Characterization of the peri-infarct zone by contrast-enhanced cardiac magnetic resonance imaging is a powerful predictor of post-myocardial infarction mortality. Circulation. 2006;114:32-9.

25. Erlinge $D$, Götberg $M$, Lang I, Holzer $M$, Noc $M$, Clemmensen $P$, Jensen $U$, Metzler B, James S, Bötker HE, Omerovic E, Engblom H, Carlsson M, Arheden H, Ostlund O, Wallentin L, Harnek J, Olivecrona GK. Rapid endovascular catheter core cooling combined with cold saline as an adjunct to percutaneous coronary intervention for the treatment of acute myocardial infarction. The CHILL-MI trial: a randomized controlled study of the use of central venous catheter core cooling combined with cold saline as an adjunct to percutaneous coronary intervention for the treatment of acute myocardial infarction. J Am Coll Cardiol. 2014;63:1857-65.

26. Atar D, Arheden H, Berdeaux A, Bonnet JL, Carlsson M, Clemmensen P, Cuvier V, Danchin N, Dubois-Randé JL, Engblom H, Erlinge D, Firat H, Halvorsen S, Hansen HS, Hauke W, Heiberg E, Koul S, Larsen Al, Le Corvoisier P, Nordrehaug JE, Paganelli F, Pruss RM, Rousseau H, Schaller S, Sonou G, Tuseth V, Veys J, Vicaut E, Jensen SE. Effect of intravenous TRO40303 as an adjunct to primary percutaneous coronary intervention for acute ST-elevation myocardial infarction: MITOCARE study results. Eur Heart J. 2015;36:112-9.

27. Moral S, Rodriguez-Palomares JF, Descalzo M, Marti G, Pineda V, Otaegui I, Garcia Del Blanco B, Evangelista A, Garcia-Dorado D. Quantification of myocardial area at risk: validation of coronary angiographic scores with cardiovascular magnetic resonance methods. Rev Esp Cardiol (Engl Ed). 2012;65:1010-7

28. Aletras AH, Kellman P, Derbyshire JA, Arai AE. ACUT2E TSE-SSFP: a hybrid method for T2-weighted imaging of edema in the heart. Magn Reson Med. 2008:59:229-35.

29. Kellman P, Aletras AH, Mancini C, McVeigh ER, Arai AE. T2-prepared SSFP improves diagnostic confidence in edema imaging in acute myocardial infarction compared to turbo spin echo. Magn Reson Med. 2007;57:891-7.

30. Payne AR, Casey M, McClure J, McGeoch R, Murphy A, Woodward R, et al. Bright-blood T2-weighted MRI has higher diagnostic accuracy than darkblood short tau inversion recovery MRI for detection of acute myocardial infarction and for assessment of the ischemic area at risk and myocardial salvage. Circ Cardiovasc Imaging. 2011:4:210-9.

31. Friedrich MG, Abdel-Aty H, Taylor A, Schulz-Menger J, Messroghli D, Dietz R. The salvaged area at risk in reperfused acute myocardial infarction as visualized by cardiovascular magnetic resonance. J Am Coll Cardiol. 2008;51:1581-7.

32. Wright J, Adriaenssens T, Dymarkowski S, Desmet W, Bogaert J. Quantification of myocardial area at risk with T2-weighted CMR: comparison with contrast-enhanced CMR and coronary angiography. JACC CardiovasC Imaging. 2009;2:825-31.

33. Giri S, Chung YC, Merchant A, Mihai G, Rajagopalan S, Raman SV, Simonetti OP. T2 quantification for improved detection of myocardial edema. J Cardiovasc Magn Reson. 2009;11:56.

34. Ugander M, Bagi PS, Oki AJ, Chen B, Hsu LY, Aletras AH, Shah S, Greiser A Kellman P, Arai AE. Myocardial edema as detected by pre-contrast $\mathrm{T} 1$ and T2 CMR delineates area at risk associated with acute myocardial infarction. JACC Cardiovasc Imaging. 2012;5:596-603.

35. Nordlund D, Klug G, Heiberg E, Koul S, Larsen TH, Hoffmann P, Metzler B, Erlinge D, Atar D, Aletras AH, Carlsson M, Engblom H, Arheden H. Multi-vendor, multicentre comparison of contrast-enhanced SSFP and T2-STIR CMR for determining myocardium at risk in ST-elevation myocardial infarction. Eur Heart J Cardiovasc Imaging. 2016;17:744-53.

36. Higgins CB, Herfkens R, Lipton MJ, Sievers R, Sheldon P, Kaufman L, Crooks LE. Nuclear magnetic resonance imaging of acute myocardial infarction in dogs: alterations in magnetic relaxation times. Am J Cardiol. 1983;52:184-8.

37. García-Dorado D, Oliveras J, Gili J, Sanz E, Pérez-Villa F, Barrabés J, Carreras MJ, Solares J, Soler-Soler J. Analysis of myocardial oedema by magnetic resonance imaging early after coronary artery occlusion with or without reperfusion. Cardiovasc Res. 1993;27:1462-9.

38. Aletras AH, Tilak GS, Natanzon A, Hsu LY, Gonzalez FM, Hoyt Jr RF, Arai AE. Retrospective determination of the area at risk for reperfused acute myocardial infarction with T2-weighted cardiac magnetic resonance imaging: histopathological and displacement encoding with stimulated echoes (DENSE) functional validations. Circulation. 2006:113:1865-70.

39. Diesbourg LD, Prato FS, Wisenberg G, Drost DJ, Marshall TP, Carroll SE, O'Neill B. Quantification of myocardial blood flow and extracellular volumes using a bolus injection of Gd-DTPA: kinetic modeling in canine ischemic disease. Magn Reson Med. 1992;23:239-53.

40. Saeed M, Wendland MF, Masui T, Higgins CB. Reperfused myocardial infarctions on T1- and susceptibility-enhanced MRl: evidence for loss of compartmentalization of contrast media. Magn Reson Med. 1994;31:31-9.

41. Arheden H, Saeed M, Higgins CB, Gao DW, Bremerich J, Wyttenbach R, Dae MW, Wendland MF. Measurement of the distribution volume of gadopentetate dimeglumine at echoplanar MR imaging to quantify myocardial infarction: comparison with 99mTc-DTPA autoradiography in rats. Radiology. 1999;211:698-708.

42. Arheden H, Saeed M, Higgins CB, Gao DW, Ursell PC, Bremerich J, Wyttenbach R, Dae MW, Wendland MF. Reperfused rat myocardium subjected to various durations of ischemia: estimation of the distribution 
volume of contrast material with echo-planar MR imaging. Radiology. 2000;215:520-8.

43. Thuny F, Lairez O, Roubille F, Mewton N, Rioufol G, Sportouch C, Sanchez I, Bergerot C, Thibault H, Cung TT, Finet G, Argaud L, Revel D, Derumeaux G, Bonnefoy-Cudraz E, Elbaz M, Piot C, Ovize M, Croisille P. Postconditioning reduces infarct size and edema in patients with ST-segment elevation myocardial infarction. J Am Coll Cardiol. 2012;59:2175-81.

44. White SK, Frohlich GM, Sado DM, Maestrini V, Fontana M, Treibel TA, Meier P, Ariti C, Davies JR, Moon JC, Yellon DM, Hausenloy DJ. Remote ischemic conditioning reduces myocardial infarct size and edema in patients with STsegment elevation myocardial infarction. JACC Cardiovasc Interv. 2015;8:178-88.

45. Josephson ME. Use of the electrocardiogram in acute myocardial infarction. N Engl J Med. 2003;348:933-40

46. Rodríguez-Palomares JF, Alonso A, Martí G, Aguadé-Bruix S, González-Alujas MT, Romero-Farina G, Candell-Riera J, García del Blanco B, Evangelista A, García-Dorado D. Quantification of myocardial area at risk in the absence of collateral flow: the validation of angiographic scores by myocardial perfusion single-photon emission computed tomography. J Nucl Cardiol. 2013:20:99-110.

\section{Submit your next manuscript to BioMed Central} and we will help you at every step:

- We accept pre-submission inquiries

- Our selector tool helps you to find the most relevant journal

- We provide round the clock customer support

- Convenient online submission

- Thorough peer review

- Inclusion in PubMed and all major indexing services

- Maximum visibility for your research

Submit your manuscript at www.biomedcentral.com/submit 